\title{
Electrochemical preparation and characterisation of bilayer films composed by Prussian Blue and conducting polymer
}

\author{
Stelian Lupu ${ }^{\text {a }}$, Constantin Mihailciuc ${ }^{\mathrm{b}}$, Laura Pigani ${ }^{\mathrm{c}}$, Renato Seeber ${ }^{\mathrm{c}, *}$, \\ Nicolae Totir ${ }^{\mathrm{d}}$, Chiara Zanardi ${ }^{\mathrm{c}}$ \\ ${ }^{a}$ Catedra de Chimie Analitica si Analiza Instrumentala, Facultatea de Chimie Industriala, Universitatea Politehnica din Bucuresti, \\ Calea Grivitei 132, 78122 Bucuresti, Romania \\ ${ }^{\mathrm{b}}$ Catedra de Chimie-fizica, Facultatea de Chimie, Universitatea Bucuresti, Bd. Regina Elisabeta 4-12, 70346 Bucuresti, Romania \\ ${ }^{\mathrm{c}}$ Dipartimento di Chimica, Università degli Studi di Modena e Reggio Emilia, via G. Campi 183, 41100 Modena, Italy \\ ${ }^{\mathrm{d}}$ Laboratorul de Electrochimie si Coroziune, Institutul de Chimie-fizica I.G. Murgulescu, Splaiul Independentei 202, 69756 Bucuresti, Romania
}

Received 30 July 2002; received in revised form 12 August 2002; accepted 13 August 2002

\begin{abstract}
Preparation and electrochemical behaviour of bilayer films consisting of iron(III) hexacyanoferrate, well known as Prussian Blue, and of poly[4,4'-bis(butylsulphanyl)-2,2'-bithiophene], on a platinum electrode, are reported. The electrochemical features of the Prussian Blue/conducting polymer bilayer system are examined in aqueous and acetonitrile solutions. Cyclic voltammetric studies show that, in acetonitrile solvent, the inner layer Prussian Blue is electroactive to some extent, though the electrochemical response of the system is mainly accounted for by poly[4,4'-bis(butylsulphanyl)-2,2'-bithiophene] outer layer. On the other hand, in aqueous solution Prussian Blue exhibits good electroactivity. Under specific experimental conditions, the individual redox behaviour of each constituent of the bilayer is evidenced in the two solvents separately, i.e., that of PB and that of poly[4,4'-bis(butylsulphanyl)-2,2'bithiophene] in aqueous and in organic solvent, respectively. However, interesting reciprocal influences are evident in the current/ potential curves recorded under conditions which are discussed. (C) 2002 Published by Elsevier Science B.V.
\end{abstract}

Keywords: Prussian Blue; Conducting polymer; Bilayer electrodes; Modified electrodes

\section{Introduction}

During the last decade, there has been a great deal of interest in electrically conducting polymers (CPs), due to their applications in electrochemical displays, in sensors, in secondary batteries, and in catalysis [1-3]. CPs can be deposited under the form of dense modifying layers on usual electrode substrates to work as electrochemical, mainly amperomeric, sensors [4-6].

On the other hand, during the last 15 years the use of metal hexacyanoferrates for the modification of electrode surfaces has attracted much interest as well [7]. Among metal hexacyanoferrates, Prussian Blue (PB), i.e., iron(III) hexacyanoferrate, is one of the most extensively studied, due to the electrochromic properties and the

\footnotetext{
${ }^{*}$ Corresponding author. Tel.: +39-059-2055027; fax: +39-059373543.

E-mail address: seeber@unimo.it (R. Seeber).
}

electrocatalytic activity and stability in aqueous solutions $[8,9]$. PB has been used in the electrocatalytic reduction of $\mathrm{CO}_{2}$ [10], $\mathrm{H}_{2} \mathrm{O}_{2}$ [11], hydrazine [12], and in the oxidation of ascorbic acid and dopamine $[13,14]$. The presence of two well distinct redox couples ascribes it selectivity with respect to the redox processes that can or cannot be electrocatalysed by an electrode modified by PB. However, the stability of PB is only satisfactory in acidic aqueous solutions, while at alkaline or neutral $\mathrm{pH}$ decomposition occurs $[15,16]$. The thermodynamics of redox systems in which $\mathrm{PB}$ is involved is of great interest when considering bilayer electrode coatings, for which it has been proposed both as the inner and as the outer phase.

The bilayer electrode coatings have been proposed since the early 1980s by Murray et al. [17] who developed a 'double redox couple system' that exhibited the interesting property of rectifying the current flow, acting similarly to a diode electronic device. Since that time, a lot of bilayer systems have been proposed, based on 
many different inorganic and organic materials [18], aimed at pursuing the original scopes, as well as at realising a variety of novel devices with various properties. In particular, bilayer systems consisting of PB films and different organic polymers have been realised. The organic polymer layer was a CP [19-22], such as polyaniline or polypyrrole, or an ion-exchange polymer, such as $\mathrm{Nafion}^{\circledR}$ [18]. It is evident that in the case that one layer is a CP and the other is $\mathrm{PB}$, the system as a whole will be affected by the conductive or insulating state of both components. This means that the response of the system will not only depend on the nature of the two layers, but also on the potential of the underlying electrode. In principle, by properly combining a suitable hexacyanoferrate, i.e., not necessarily iron hexacyanoferrate, with a suitable $\mathrm{CP}$, a wide choice of possible selectivity in the correspondence to different potentials can be realised. It is, however, very important to test carefully the experimental conditions, since formation of mixed-valence states could vanish any project based on strict thermodynamic arguments $[23,24]$.

In the recent past, we worked extensively on S-alkyl substituted polythiophenes, in particular on the poly[4,4'bis(butylsulphanyl)-2,2'-bithiophene] (PBSBT) [25-31], i.e., a $\mathrm{CP}$ that proved to posses unique stability properties, from both a mechanical and a chemical point of view. This allowed us to perform time-consuming measurements $[28,29]$ devoted to the electrochemical characterisation of the $\mathrm{CP}$ coating. In two recent papers, we reported the capabilities of PBSBT modified conventional-size and microelectrodes in analyses performed in aqueous media at neutral $\mathrm{pH}$, by linear sweep (LSV) and differential pulse voltammetry (DPV) [30,31].

Aiming at obtaining stable bilayer PB-CP films, in view of the advantages offered by PBSBT, we have studied preparation and electrochemical features of a PB/PBSBT bilayer coating deposited on a platinum electrode. The $\mathrm{PB} / \mathrm{PBSBT}$ coverage is prepared electrochemically by a two-step method. We first electrodeposited a PB layer onto a platinum electrode in aqueous solution. In the second step, the electrochemical deposition of PBSBT film over the $\mathrm{Pt} / \mathrm{PB}$ modified electrode was carried out in an organic medium. Hereafter, the electrochemical behaviour and the stability of the bilayer coating are studied in both organic and aqueous solutions. The very good adherence of PBSBT to PB, in addition to the well known stability of the PB film on metal electrodes, allowed quite a stable bilayer coating system to be realised.

\section{Experimental}

\subsection{Chemicals}

All chemicals: $\mathrm{FeCl}_{3}$ (Merck), $\mathrm{K}_{3}\left[\mathrm{Fe}(\mathrm{CN})_{6}\right]$ (Feinbiochemica), $\mathrm{HCl}$ (Aristar), and $\mathrm{KCl}$ (Carlo Erba, RPE) were used as received. 4,4'-bis(butylsulphanyl)-2,2'-bithiophene (BSBT) was synthesised as described previously [32]. Tetrabutylammonium hexafluorophosphate supporting electrolyte $\left(\mathrm{TBAPF}_{6}\right)$ from Fluka, puriss., acetonitrile (AN) solvent from Aldrich, 99.8\%+ pure, anhydrous, packaged under nitrogen were used as received. Doubly distilled water was always used to prepare aqueous solutions for electrochemical use. In order to de-aerate the solutions for electrochemical tests, nitrogen $(99.99 \%+$ purity) was bubbled through, for $20 \mathrm{~min}$ before the experiments; nitrogen flowed continuously over the sample solution during the measurements.

\subsection{Apparatus and electrodes}

The electrochemical experiments were carried out with an Autolab PGSTAT12 potentiostat-function generator electrochemical instrument (Ecochemie, Utrecht, The Netherlands), using a single-compartment, three-electrode cell. A 3-mm diameter Pt disk electrode (Metrohm, Herisau, Switzerland) was the working electrode, a saturated calomel electrode (SCE, Amel, Milan, Italy) was the reference electrode, and a glassy carbon rod (Metrohm) was the auxiliary electrode. All potential values, both in organic and in aqueous media, are referred to SCE. Before each electrochemical test the surface of the working electrode was polished subsequently with 1 and $0.3 \mu \mathrm{m}$ alumina powder to a mirror finish, dipped into an ultrasonic bath for $5 \mathrm{~min}$, and then rinsed with doubly distilled water.

Optical and electron microscopy were used in order to ascertain the occurrence of complete electrode coverage both by the inner and by the other layer and to check their uniform structure.

\section{Results and discussion}

\subsection{Pt/PB modified electrode}

PB was deposited onto the electrode by potentiostatic method at a potential value of $+0.40 \mathrm{~V}$, from an aqueous solution containing $2 \times 10^{-3} \mathrm{M} \mathrm{K}_{3}\left[\mathrm{Fe}(\mathrm{CN})_{6}\right]$ and $2 \times 10^{-3} \mathrm{M} \mathrm{FeCl}_{3}$ in $0.1 \mathrm{M} \mathrm{KCl}+0.01 \mathrm{M} \mathrm{HCl}$. The electrolysis was prolonged for $60 \mathrm{~s}$. After deposition, the modified electrodes were rinsed with doubly distilled water and immersed into a solution containing $0.1 \mathrm{M}$ $\mathrm{KCl}$ and $0.01 \mathrm{M} \mathrm{HCl}$, where the electrode potential was cycled between 0.00 and $+1.00 \mathrm{~V}$ at a scan rate of $0.05 \mathrm{~V} \mathrm{~s}^{-1}$, until a stable voltammetric response was obtained.

\subsubsection{Electrochemical behaviour of Pt/PB modified electrode in aqueous medium}

A typical cyclic voltammogram recorded on a $\mathrm{Pt} /$ PB modified electrode, obtained as described above, is 
shown in Fig. 1(a). The Everitt's Salt (ES) is obtained by reduction of $\mathrm{PB}$, while oxidation gives Berlin Green (BG) [33]. As it is well known [15], the signals in Fig. 1(a) are ascribed to the following processes:

- peak system at ca. $+0.2 \mathrm{~V}$ :

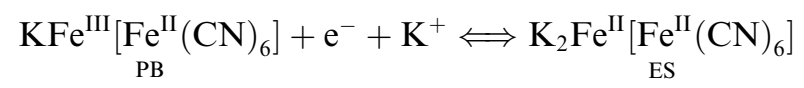

- peak system at ca. $+0.9 \mathrm{~V}$ :

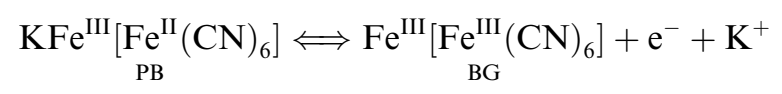

The linear dependence on the potential scan rate of the forward cathodic peak current intensities of the peak system at ca. $+0.20 \mathrm{~V}$ up to $0.10 \mathrm{~V} \mathrm{~s}^{-1}$ and the shape of the cathodic-anodic response supports the surface nature of the electrode process expressed by Eq. (1); accordingly, the peak potential separation for the system at ca. $+0.20 \mathrm{~V}$ is equal to ca. $20 \mathrm{mV}$ at the scan rate of $0.05 \mathrm{~V} \mathrm{~s}^{-1}$ and progressively increases at increasing the scan rate, no ohmic drop compensation being applied. The value of the peak potential separation indicates that $\mathrm{PB}$ adheres strongly to the electrode surface, that the PB layer is thin, and that its conductivity is high.

The excellent stability of the modified electrode over 100 repetitive scans between 0.00 and $+1.00 \mathrm{~V}$ in $0.1 \mathrm{M}$ $\mathrm{KCl}$ and $0.01 \mathrm{M} \mathrm{HCl}$ solution should be emphasised. Only a slight decrease of ca. $5 \%$ of both anodic and cathodic peak currents of the system at less positive potential is observed. The stability of the peak system at more positive potentials is even higher, and only a decrease of the peak currents of $2 \%$ is correspondingly observed.

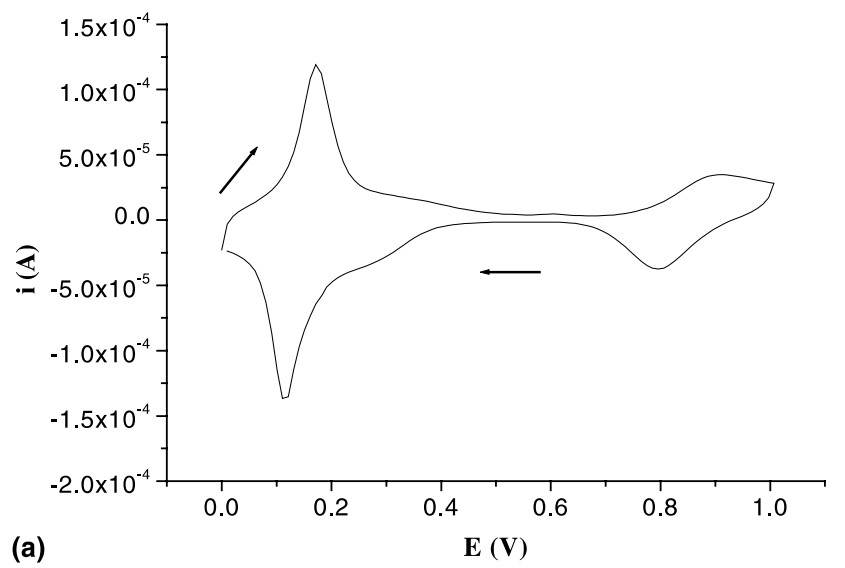

3.1.2. Electrochemical behaviour of Pt/PB modified electrode in organic media

When the PB modified electrode is transferred into AN solution only containing $0.1 \mathrm{M} \mathrm{TBAPF}_{6}$, the $\mathrm{PB} / \mathrm{ES}$ redox process is prevented from occurring significantly, while the signal corresponding to the $\mathrm{PB} / \mathrm{BG}$ redox couple is well detectable. This is evident from the dotted line voltammetric curve in Fig. 1(b). On the other hand, when the same solution is added with $0.01 \mathrm{M} \mathrm{KClO}_{4}$, the PB film exhibits an interesting change in the redox behaviour: the PB/ES peak system is well developed, though lower than in aqueous medium, showing a peak potential separation $\left(\Delta E_{\mathrm{p}}\right)$ of $60 \mathrm{mV}$ at $0.02 \mathrm{~V} \mathrm{~s}^{-1}$ potential scan rate, and the $\mathrm{PB} / \mathrm{BG}$ peak system is comparable in height with that recorded in aqueous medium, exhibiting a $\Delta E_{\mathrm{p}}$ value of $70 \mathrm{mV}$ at $0.02 \mathrm{~V} \mathrm{~s}^{-1}$ potential scan rate (solid line in Fig. 1(b)). However, the low solubility of $\mathrm{KClO}_{4}$ salt in AN prevents from making reference to comparable experimental conditions. An explanation to the different behaviour can be found in a lower exchange rate of $\mathrm{K}^{+}$ions between the PB film and the solution, also due to their lower concentration, which affects both the reversibility degree of the redox processes and the height of the relevant responses.

Therefore, the highly reversible behaviour of the $\mathrm{PB} /$ ES system in aqueous solution turns into a quasireversible, however well detectable response in AN solution containing $\mathrm{KClO}_{4}$, despite a 10-fold lower concentration. The reversibility degree of the $\mathrm{PB} / \mathrm{BG}$ system is comparable in the two quite different media.

\subsection{Pt/PB/PBSBT modified electrode}

The outer polymer electrode coating was prepared by direct electrochemical polymerisation-deposition on the $\mathrm{Pt} / \mathrm{PB}$ electrode, carried out in a solution containing $5 \times 10^{-3} \mathrm{M}$ BSBT monomer and $0.1 \mathrm{M}$ TBAPF $_{6}$

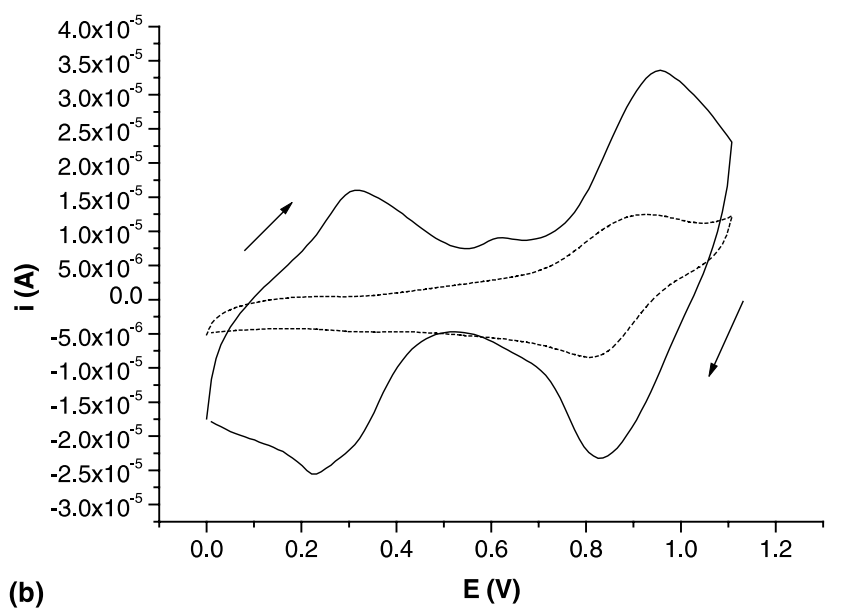

Fig. 1. Cyclic voltammogram of $\mathrm{Pt} / \mathrm{PB}$ electrode in (a) aqueous solution containing $0.1 \mathrm{M} \mathrm{KCl}, 0.01 \mathrm{M} \mathrm{HCl}_{\text {; }}$ (b) dotted line: $0.1 \mathrm{M}$ TBAPF , AN solution; solid line: $0.1 \mathrm{M} \mathrm{TBAPF}_{6}, 0.01 \mathrm{M} \mathrm{KClO}_{4}$, AN solution. $0.05 \mathrm{~V} \mathrm{~s}^{-1}$ potential scan rate. 


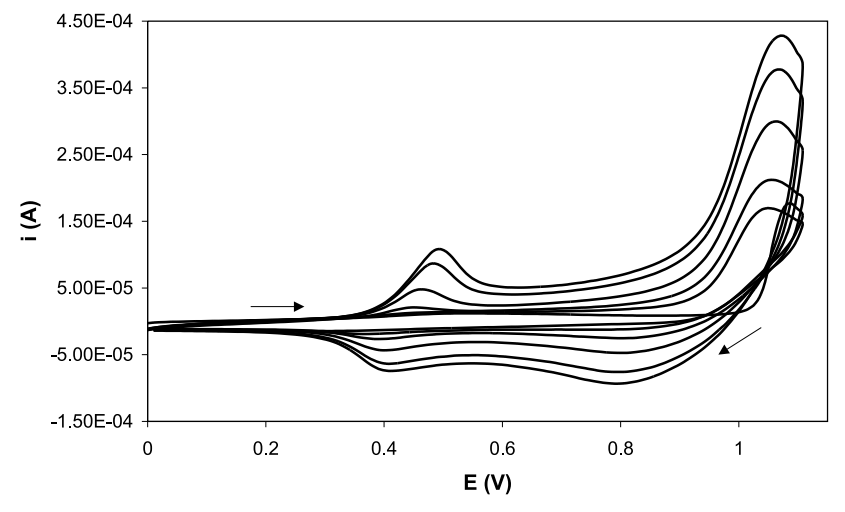

Fig. 2. Polymerisation of $5 \mathrm{mM}$ BSBT on $\mathrm{Pt} / \mathrm{PB}$ modified electrode in $0.1 \mathrm{M} \mathrm{TBAPF}_{6}, \mathrm{AN}$ solution. $0.05 \mathrm{~V} \mathrm{~s}^{-1}$ potential scan rate.

supporting electrolyte in AN solvent. The PBSBT polymer film was grown by cycling the electrode potential in the range from 0.00 to $+1.05 \mathrm{~V}$, at a scan rate of $0.05 \mathrm{~V} \mathrm{~s}^{-1}$ (see Fig. 2). The occurrence of monomer oxidation, which consists of a reversible charge transfer and is necessary in order to start polymerisation as well as to sustain chain propagation, occurs at potentials at which the thermodynamically stable form is BG, i.e., a species capable to induce electrocatalytic oxidation.

The voltammograms recorded during the polymer growth are similar to those observed in the case of the BSBT directly polymerised on bear Pt electrode. However, the peak current values are smaller, due to the low electroactivity of $\mathrm{PB}$ films in acetonitrile solution. By addition of $0.01 \mathrm{M} \mathrm{KClO}_{4}$, the polaron and bipolaron formation peaks are better defined and the peak current values are higher.

It is noteworthy that the PB coating allows for deposition of the PBSBT film over, despite the fact that PB exhibits a very much depressed electroactivity in organic media.

\subsubsection{Electrochemical behaviour of $P t / P B / P B S B T$ in aqueous media}

In order to analyse the effect of the presence of one layer on the other one under different experimental conditions, the electrochemical behaviour of the $\mathrm{Pt} / \mathrm{PB} /$ PBSBT electrode has been studied in both organic and aqueous solutions, using different supporting electrolytes. Fig. 3 - solid line - shows the cyclic voltammetric response of the bilayer modified electrode in $0.1 \mathrm{M} \mathrm{KCl}$, $0.01 \mathrm{M} \mathrm{HCl}$ aqueous solution; comparison with the response of $\mathrm{Pt} / \mathrm{PB}$ in a similar solution is possible by looking at Fig. 1(a). With an outer layer consisting of PBSBT, the redox activity of the PB film is partially hindered, but the peak system corresponding to the $\mathrm{PB} /$ ES redox couple is still well detectable: the difference between anodic and cathodic peak potentials is ca. 180 $\mathrm{mV}$ at a scan rate of $0.05 \mathrm{~V} \mathrm{~s}^{-1}$, i.e., the charge transfer is quasi-reversible. The formal potential for the redox couple $\mathrm{PB} / \mathrm{ES}$, evaluated approximately as the half-sum of the anodic and cathodic peak potential values, is equal to $+0.13 \mathrm{~V}$. Significantly, this value is fairly similar to that of the $\mathrm{PB} / \mathrm{ES}$ system computed on a $\mathrm{Pt} / \mathrm{PB}$ electrode, in aqueous solution, which is $+0.14 \mathrm{~V}$. This result indicates that the thermodynamics of the $\mathrm{PB} / \mathrm{ES}$ redox system is unaffected by the presence of the outer PBSBT layer. Furthermore, the current peaks relative to the redox switching from $\mathrm{PB}$ to $\mathrm{BG}$ and vice versa are hardly detectable, possibly due to the proximity to the solvent oxidation. The different character of the redox process involving $\mathrm{PB}$ to $\mathrm{BG}$ oxidation could be due to the fact that, at those potentials, the PBSBT polymer is positively charged and opposes the exchange of $\mathrm{K}^{+}$ions between the electrolyte solution and the PB layer, which is a necessary requirement in order that reaction (2) occurs. Finally, the response due to polaron formation on the outer PBSBT layer is well detectable at ca. +0.5 $\mathrm{V}$. It is difficult, in this case, to conclude if the detection of the signal ascribed to polaron formation is due to the formation of significant quantities of $\mathrm{BG}$ form at that potential, which induces electrocatalytic p-doping or, rather, to the conductive character of $\mathrm{PB}$ due to a mixed-valence ES/PB phase $[22,23]$. The formation of such a phase can be ascribed to the necessarily slow charge propagation through the inorganic layer. The formation of mixed-valence states is plausible when working under potentiodynamic conditions with a relatively high value of the potential scan rate, i.e., quite far from equilibrium.

The stability of the PB/PBSBT bilayer coating in aqueous solution has also been tested. Fig. 3 - broken line - reports a cyclic voltammogram recorded on the $\mathrm{Pt} / \mathrm{PB} / \mathrm{PBSBT}$ system in aqueous solution after $60 \mathrm{~min}$ immersion under 'open circuit' conditions. Only a barely appreciable decrease of the cathodic peak current relative to PB to ES reduction can be observed. On the other

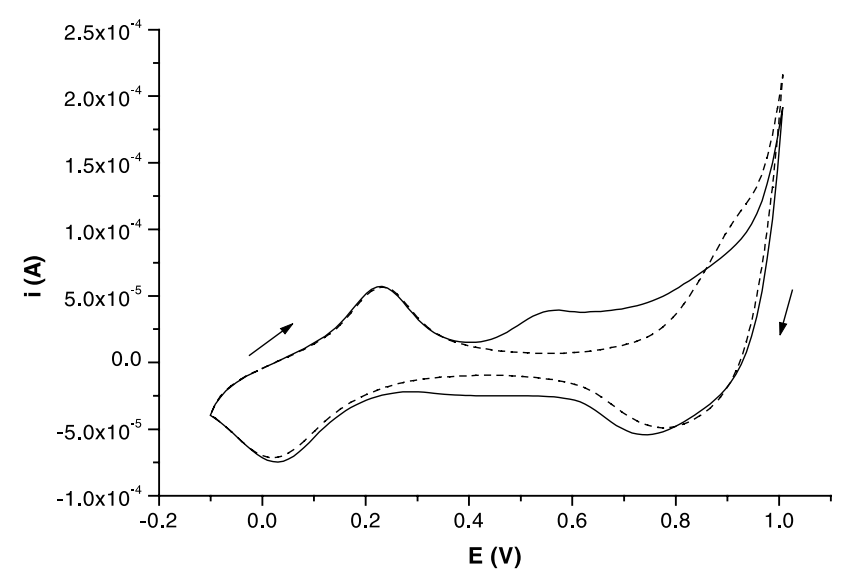

Fig. 3. Cyclic voltammograms of $\mathrm{Pt} / \mathrm{PB} / \mathrm{PBSBT}$ in aqueous solution containing $0.1 \mathrm{M} \mathrm{KCl}, 0.01 \mathrm{M} \mathrm{HCl}$ (solid line) and after $60 \mathrm{~min}$ dipping (dashed line). $0.05 \mathrm{~V} \mathrm{~s}^{-1}$ potential scan rate. 


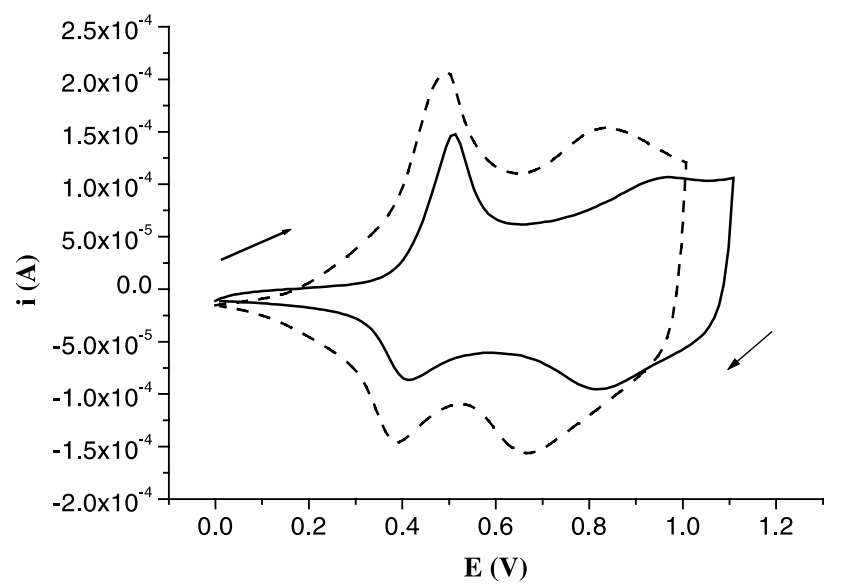

Fig. 4. Cyclic voltammograms of $\mathrm{Pt} / \mathrm{PB} / \mathrm{PBSBT}$ in $0.1 \mathrm{M} \mathrm{TBAPF}_{6}, \mathrm{AN}$ solution (solid line) and in $0.1 \mathrm{M} \mathrm{TBAPF}_{6}, 0.01 \mathrm{M} \mathrm{KClO}_{4}, \mathrm{AN}$ solution (broken line). $0.05 \mathrm{~V} \mathrm{~s}^{-1}$ potential scan rate.

hand, the response due to polaron formation on the outer PBSBT layer is not detectable anymore. This is, however, a problem already observed for the same polymer in aqueous solution, preventing us from speculating about the actual conductivity of the inner PB layer under such experimental conditions.

\subsubsection{Electrochemical behaviour of $P t / P B / P B S B T$ in organic media}

When the bilayer coated electrode is transferred into an AN solution containing $0.1 \mathrm{M} \mathrm{TBAPF}_{6}$ immediately $^{-}$ after preparation, the typical cyclic voltammetric response of the PBSBT film alone is observed (solid line in Fig. 4). Two sets of peaks are present at ca. $+0.50 \mathrm{~V}$ and $+0.90 \mathrm{~V}$, corresponding to polaron and bipolaron formation and relevant neutralisation, respectively. These values of the peak potentials are fairly coincident with those observed for the PBSBT film alone in AN solution containing $0.1 \mathrm{M} \mathrm{TBAPF}_{6}$ [27].

A similar behaviour of the $\mathrm{Pt} / \mathrm{PB} / \mathrm{PBSBT}$ modified electrode is observed in $\mathrm{AN}$ solution containing $0.1 \mathrm{M}$ $\mathrm{TBAPF}_{6}$ and $0.01 \mathrm{M} \mathrm{KClO}_{4}$ (dotted line in Fig. 4). The sets of peaks corresponding to the polaron formation are observed also in this case; however, they are both significantly higher, in agreement with the supply of $\mathrm{K}^{+}$ ions from the solution to the underlying PB layer. Furthermore, the peaks corresponding to the bipolaron formation and neutralisation are better defined and located at less positive potential values. This constitutes the most remarkable change in the voltammogram recorded after addition of $\mathrm{K}^{+}$ions to the solution. It is reasonable to suggest that the BG species that forms at slightly less positive potentials acts as a catalyst with respect to the polaron to bipolaron conversion, which is affected by charge transfer overvoltage. As to the inner layer, only when $\mathrm{K}^{+}$ions are added to the solution, though at a very low concentration, a small signal attributable to the PB/ES system may be identified, suggesting that $\mathrm{PB}$ undergoes redox conversion according to (1) under these conditions.

\section{Conclusions}

The work described in this paper has been planned in order to check the possibility of preparing and working with a novel bilayer system consisting of an inner inorganic phase that attracted so many interest as a redox mediator and that includes two distinct redox couples, and of an outer phase consisting of a CP that proved us to posses lot of interesting properties. The system is studied under the potentiodynamic conditions that are usually proper of electroanalytical tests. Studies devoted to better define the physico-chemical properties of the system and of the PB - CP interface will deserve further attention, requiring potentiostatic and faradic impedance measurements, in order to analyse the system under steady-state and equilibrium conditions. Furthermore, our tests have been carried out by using layer formed under reproducible conditions; however, we did not care of fixing either the single thickness or the ratio between them. Precious information can be drawn out from systematic tests at varying systematically the thickness parameters.

\section{Acknowledgements}

S.L. appreciates a fellowship from the Romanian Ministry of National Education (MEN) supporting a six months stay at the Department of Chemistry, University of Modena.

\section{References}

[1] J. Heinze, in: Topics in Current Chemistry, vol. 152, Springer, Berlin, 1990.

[2] M.E.G. Lyons (Ed.), Electroactive Polymer Electrochemistry, Part 1. Fundamentals, Part 2. Methods and Applications, Plenum Press, New York, 1994.

[3] J. Roncali, J. Mater. Chem. 9 (1999) 1875, and references therein.

[4] R.W. Murray, in: A.J. Bard (Ed.), Electroanalytical Chemistry, vol. 13, Marcel Dekker, New York, 1984, p. 191.

[5] R.W. Murray (Ed.), Molecular Design of Electrode Surfaces, vol. 22, Wiley, New York, 1992.

[6] G. Inzelt, in: A.J. Bard (Ed.), Electroanalytical Chemistry, vol. 18, Marcel Dekker, New York, 1994.

[7] J.A. Cox, R.K. Jaworski, P.J. Kulesza, Electroanalysis 3 (1991) 869.

[8] K. Itaya, I. Uchida, V.D. Neff, Acc. Chem. Res. 19 (1986) 162.

[9] A.A. Karyakin, Electroanalysis 13 (2001) 813.

[10] K. Ogura, N. Endo, M. Nakayama, H. Ootsuka, J. Electrochem. Soc. 106 (1984) 3423.

[11] A.A. Karyakin, E.E. Karyakina, L. Gorton, Talanta 43 (1996) 1597. 
[12] U. Scharf, E. Grabner, Electrochim. Acta 41 (1996) 233.

[13] N. Totir, S. Lupu, E.M. Ungureanu, M. Giubelean, A. Stefanescu, Rev. Roum. Chim. (in press).

[14] N. Totir, S. Lupu, E.M. Ungureanu, N. Iftimie, Rev. Chim. (Bucharest, English Edition) 2 (2001) 23.

[15] K. Itaya, H. Akahoshi, S. Toshima, J. Electrochem. Soc. 129 (1982) 1498.

[16] R. Koncki, O.S. Wolfbeis, Anal. Chem. 70 (1998) 2544.

[17] H.D. Abruña, P. Denisevich, M. Umaña, T.J. Meyer, R.W. Murray, J. Am. Chem. Soc. 103 (1981) 1.

[18] R.J. Mortimer, J. Electroanal. Chem. 397 (1995) 79, and references therein.

[19] K. Ogura, N. Endo, M. Nakayama, H. Ootsuka, J. Electrochem. Soc. 142 (1995) 4026.

[20] W. Lu, G.G. Wallace, A.A. Karyakin, Electroanalysis 10 (1998) 472.

[21] K. Ogura, K. Nakaoka, M. Nakayama, J. Electroanal. Chem. 486 (2000) 119 .

[22] O. Somani, S. Radhakrishnan, Mater. Chem. Phys. 76 (2002) 15.

[23] B.J. Feldman, R.W. Murray, Inorg. Chem. 26 (1987) 1702.
[24] P.J. Kulesza, Z. Galus, J. Electroanal. Chem. 323 (1992) 261.

[25] D. Iarossi, A. Mucci, L. Schenetti, R. Seeber, F. Goldoni, M. Affronte, F. Nava, Macromolecules 32 (1999) 1390.

[26] H. Ding, L. Pigani, R. Seeber, C. Zanardi, J. New Mater. Electrochem. Syst. 3 (2000) 337.

[27] B. Ballarin, F. Costanzo, F. Mori, A. Mucci, L. Pigani, L. Schenetti, R. Seeber, D. Tonelli, C. Zanardi, Electrochim. Acta 46 (2001) 881.

[28] H. Ding, Z. Pan, L. Pigani, R. Seeber, C. Zanardi, Electrochim. Acta 46 (2001) 2721.

[29] H. Ding, Z. Pan, L. Pigani, R. Seeber, J. New Mater. Electrochem. Syst. 4 (2001) 63.

[30] S. Lupu, A. Mucci, L. Pigani, R. Seeber, C. Zanardi, Electroanalysis 14 (2002) 519.

[31] S. Lupu, L. Pigani, R. Seeber, C. Zanardi, Electroanalysis (in press).

[32] U. Folli, F. Goldoni, D. Iarossi, A. Mucci, L. Schenetti, J. Chem. Res. (1996) 69.

[33] K. Itaya, T. Ataka, S. Toshima, J. Am. Chem. Soc. 104 (1982) 4767. 